\title{
Tuberculosis en trabajadores de salud: Estudio epidemiológico y clínico en el Hospital Nacional Hipólito Unanue
}

Tuberculosis in health workers: clinical and epidemiological study in Hospital Nacional Hipólito Unánue

Mayron Nakandakari 1,a, Dyanne De la Rosa ${ }^{1, a}$, Jorge Gutierrez ${ }^{2, b}$, Walter Bryson ${ }^{3, c}$

RESUMEN

Objetivo: Describir las características epidemiológicas y clínicas de los trabajadores de salud del Hospital Nacional Hipólito Unanue (HNHU) con diagnóstico de Tuberculosis (TBC) entre el 2006 y 2013. Material y métodos: Estudio observacional, descriptivo, longitudinal, retrospectivo, realizado en el HNHU de categoría III-1. La población estudiada estuvo constituida por los trabajadores de salud con diagnóstico de Tuberculosis entre el 2006 y el 2013. La muestra fue todo el universo. La técnica fue de documentación. Se utilizó estadística descriptiva y los programas Microsoft Excel 2010 y SPSS v 19. Resultados: Se identificaron 56 trabajadores de Salud con diagnóstico de TBC, 4 fueron BK (+++) y uno falleció. La frecuencia de los casos tuvo una tendencia a disminuir desde el 2008, con su valor más bajo en el 2012. Conclusiones: La mayoría fueron diagnosticados en el 2007 y 2013, médicos residentes, BK negativo, con TBC pulmonar sensible y del Servicio de Hospitalización de Especialidades.

PALABRAS CLAVE: Tuberculosis, personal de salud, infección hospitalaria. (Fuente: DeCS BIREME).

\section{SUMMARY}

Objective: To describe clinical and epidemiological features of tuberculosis (TBC) among health care workers (HCW) at Hospital Nacional Hipólito Unánue (HNHU) from 2006 to 2013. Methods: Observational study conducted at HNHU (category III-1 hospital). All HCW with the diagnosis of TBC within the study period were included. Descriptive statistics were carried-out using Microsoft Excel 2010 and SPSS v 19. Results: 56 HCW were identified, 4 had sputum smears 4+plus and one died. TBC rates decreased since 2008 with a nadir in 2012. Conclusions: Most of the HCW were diagnosed between 2007 and 2013, being mostly medical residents with negative sputum smears and drug sensitive TBC from the specialty hospitalization services.

KEYWORDS: Tuberculosis, health personnel, cross infection (Source: MeSH NLM).

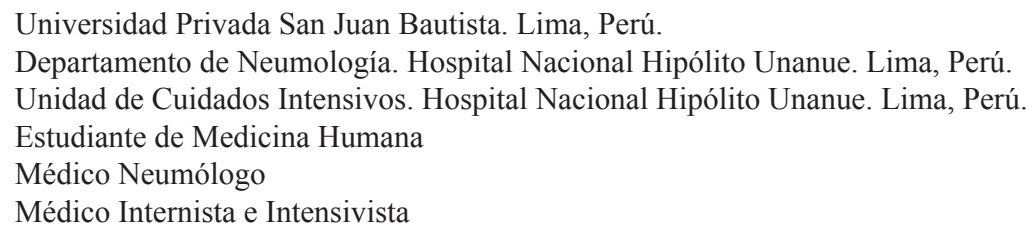




\section{INTRODUCCIÓN}

La Tuberculosis (TBC) es una enfermedad infectocontagiosa, producida por el Mycobacterium tuberculosis, causante de más de 2,5 millones de fallecimientos cada año en el mundo (1). Eso significa la pérdida de una vida cada 15 segundos. Así mismo, cada enfermo con TBC infecta de 10 a 15 personas por año, en promedio, siendo tres cuartos de todos los infectados adultos jóvenes (2).

Globalmente hay un 3\% de incremento de casos nuevos cada año. Según el Ministerio de Salud del Perú (MINSA) (3), cada hora, cuatro ciudadanos peruanos son diagnosticados de TBC. Cada año se reportan cerca de 35000 ciudadanos con TBC activa, de los cuales cerca del 7 a $10 \%$ son producidos por cepas mutantes resistentes a múltiples drogas. Todo ello conlleva a que el Perú figure en el séptimo puesto en el mundo entre los países con mayor problema de TBC multidrogorresistente (MDR) y cuarto en número absoluto estimado de casos de personas con TBC MDR (4).

Esta realidad peruana condiciona que se eleve 40 veces más el riesgo de una enfermedad tuberculosa en el personal de salud que en la población general (5). Amenazando así al personal hospitalario que trabaja en condiciones no adecuadas tales como el hacinamiento en áreas críticas de algunos servicios, sin la bioseguridad que garantice no contagiarse; situación laboral muy común en todos los hospitales del Perú (6).

Evidencia para esto son las cifras oficiales del MINSA (7): 170 casos de TBC en trabajadores de salud en el año 2012, de los cuales 67 fueron TBC MDR. Todo ello genera un problema de Salud Pública y ocupacional grave que registra anualmente en el Perú una tasa de incidencia oscilante entre 69 y 5 780 trabajadores de salud con TBC por cada 100000 habitantes.

En este contexto, debido a la innegabilidad de que el trabajador hospitalario se encuentra totalmente expuesto a contraer TBC $(8,9)$; se realizó el estudio cuyo objetivo general fue describir las características epidemiológicas y clínicas de los trabajadores de salud de un hospital general con diagnóstico de TBC.

\section{MATERIAL Y MÉTODOS}

Estudio de tipo observacional, descriptivo, transversal y retrospectivo. Se realizó en el Hospital
Tabla 1. Características bio-ocupacionales del personal de salud del Hospital Nacional Hipólito Unanue diagnosticados de Tuberculosis, 2006-2013.

\begin{tabular}{lll}
\hline Características & n & $\%$ \\
\hline
\end{tabular}

\section{Sexo}

Femenino

$33 \quad 58,9$

Masculino

2341,1

Antecedente de TBC previa
No

Sí

\section{Condición Laboral}

Contratado

Nombrado

Residente

Interno

Otros

\section{Grupo Ocupacional}

Médico Residente

Técnico de Enfermería

Médico

Auxiliar Nutrición

Interno de Medicina

Personal de limpieza

Administrativo

Mantenimiento

Enfermera

Interno otras especialidad

Tecnólogo Médico

Técnico de Farmacia

Interno Odontología

Obstetríz

$\begin{array}{rr}51 & 91,1 \\ 5 & 8,9\end{array}$

$27 \quad 48,2$

$8 \quad 14,3$

$7 \quad 12,5$

$5 \quad 8,9$

$9 \quad 16,1$
4,3
2,5
8,9
6,1

14,3

$8 \quad 14,3$

$7 \quad 12,5$

$5 \quad 8,9$

$4 \quad 7,1$

$4 \quad 7,1$

$4 \quad 7,1$

$4 \quad 7,1$

$4 \quad 7,1$

35,4

23,6

$1 \quad 1,8$

$1 \quad 1,8$

$1 \quad 1,8$
Nacional Hipólito Unanue (HNHU) de categoría III-1 perteneciente a la Dirección de Salud IV Lima Este y al MINSA.

La población estudiada fueron los trabajadores de salud con diagnóstico de tuberculosis entre el 2006 y el 2013, atendidos en el Programa de Control de Tuberculosis (PCT) del HNHU. Los criterios de inclusión fueron: ser trabajadores con diagnóstico de TBC activa en el periodo de estudio, haber laborado 
en el hospital por más de 30 días y tener historia clínica completa. Se excluyeron a los trabajadores diagnosticados en el PCT del HNHU, pero con tratamiento antiTBC en otro sanatorio.

El tamaño muestral fue considerado como todo el universo de trabajadores de salud que ingresó al PCT del HNHU entre el período de 2006- 2013.

La variable principal de nuestro estudio fue "características epidemiológicas y clínicas de los trabajadores de salud con TBC", que se determinó por $(10,11)$ : Número de casos por año, sexo, antecedente de TBC previa, condición laboral, grupo ocupacional, resultados de baciloscopía al momento del diagnóstico, localización de la enfermedad, tipo de TBC, condición de tratamiento, condición de alta y servicio del hospital donde laboraba.

Para la obtención de la información se adaptó una hoja de recolección de datos con 11 ítems específicos para este estudio. Luego se procedió a la recolección de la información a partir de las historias clínicas digitadas en las bases de dato de la Oficina de Estadística e Informática del HNHU.

Los datos fueron ingresados a una base diseñada para el estudio en los paquetes estadísticos Microsoft Excel 2010 y SPSS v. 19 para Windows.

El trabajo fue aprobado para su realización por el Comité de Ética en Investigación del HNHU.

\section{RESULTADOS}

Durante el periodo de estudio 2006-2013 se identificaron 56 trabajadores de Salud con diagnóstico de TBC pertenecientes al PCT del HNHU.

El gráfico 1 sobre la frecuencia de casos de TBC en el personal de salud muestra un primer incremento de los casos en el 2007, una tendencia a disminuir desde el 2008 teniendo el valor más bajo en el 2012. No obstante, en el 2013 ocurrió un incremento súbito llegando a 10 casos registrados.

En la tabla 1, lo más relevante es que el 33,9\% de los casos de TBC ocurrió en médicos, médicos residentes e internos de Medicina, evidenciando que son los más expuestos por estar en mayor contacto con los pacientes. Así mismo, se encontró que sólo el 8,9\% tuvo historia de TBC antes del 2006, mismos que se reinfectaron y volvieron a desarrollar TBC durante el periodo de estudio.

Los resultados de baciloscopía, localización de la enfermedad y esquema de tratamiento, se muestran en la tabla 2. En la mitad el BK fue negativo, siendo el diagnóstico radiológico y clínico. La localización pulmonar fue la más frecuente y el $94,6 \%$ de los casos recibió Esquema I de tratamiento.

El 53,6\% tuvo TBC Pulmonar sensible; el 14,3\% de los casos, fueron TBC MDR por la prueba de Genotype ${ }^{\circledR}$ MTDBR plus realizada en el Instituto Nacional de Salud (Tabla 3).

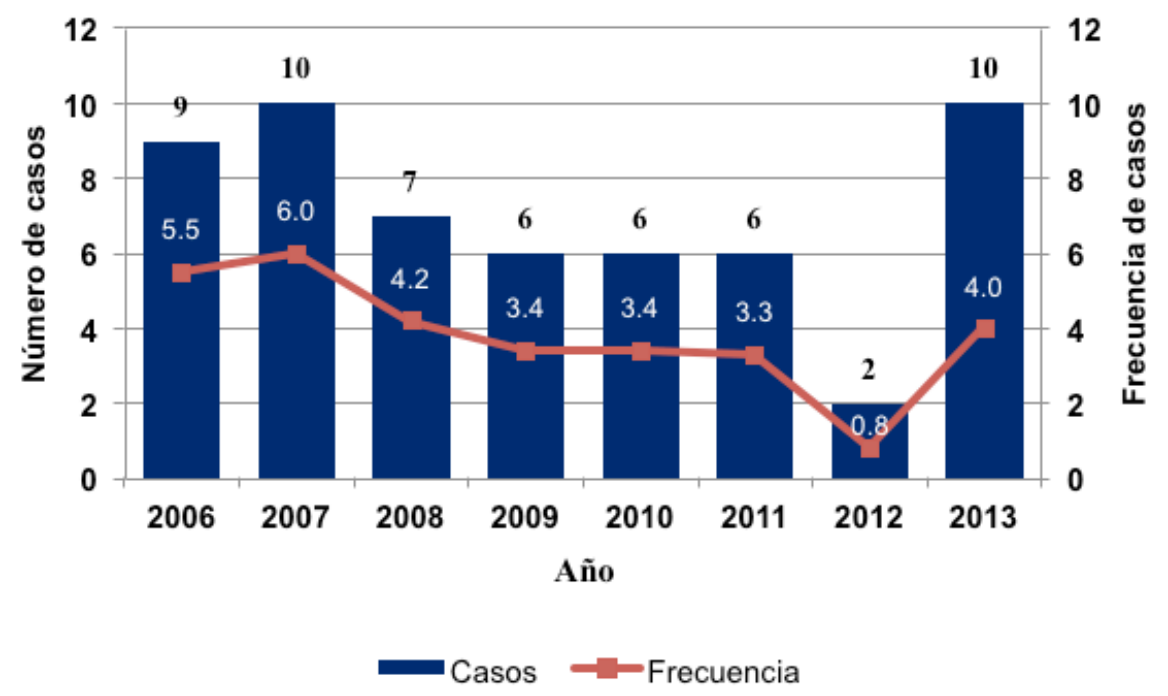

Gráfico 1. Casos de Tuberculosis en el personal de salud del Hospital Nacional Hipólito Unanue, 2006-2013. 
Tabla 2. Condiciones Diagnósticas, de Tratamiento y de alta del personal de salud del Hospital Nacional Hipólito Unanue, 2006-2013.

\begin{tabular}{lcr}
\hline Características Diagnósticas & $\mathbf{n}$ & $\mathbf{\%}$ \\
\hline Baciloscopía diagnóstica & 28 & 50,0 \\
BK (-) & 24 & 42,9 \\
BK (+) & 4 & 7,1 \\
BK (+++) & & \\
Localización de la enfermedad & 44 & 78,6 \\
$\quad$ Pulmonar & 12 & 21,4 \\
$\quad$ Extrapulmonar & & \\
Esquema de tratamiento & 53 & 94,6 \\
$\quad$ Esquema I & 1 & 1,8 \\
$\quad$ Esquema II & 2 & 3,6 \\
$\quad$ Esquema III-MDR & & \\
Condición de Alta & 46 & 82,1 \\
$\quad$ Curado & 9 & 16,1 \\
$\quad$ En tratamiento & 1 & 1,8 \\
$\quad$ Fallecido & & \\
\hline
\end{tabular}

Tabla 3. Tipos de Tuberculosis (TBC) en el personal de salud del Hospital Nacional Hipólito Unanue, 2006-2013.

\begin{tabular}{lcc}
\hline Tipos de TBC & $\mathbf{n}$ & $\mathbf{\%}$ \\
\hline TBC Pulmonar sensible & 30 & 53,6 \\
TBC Pulmonar MDR & 8 & 14,3 \\
TBC Pleural & 7 & 12,5 \\
TBC Pulmonar + Recaída & 5 & 8,9 \\
TBC Meníngea & 3 & 5,4 \\
TBC Ganglionar & 2 & 3,6 \\
TBC Paucibacilar & 1 & 1,8 \\
TOTAL & 56 & 100,0 \\
\hline
\end{tabular}

La distribución de los casos de TBC según los diferentes servicios hospitalarios en los que laboraban, se muestra en la tabla 4; se identificó que los más afectados fueron los que laboraban en los servicios de Hospitalización de Especialidades seguidos por los del servicio de Mantenimiento y Limpieza del hospital.

\section{DISCUSIÓN}

Es definitivo que el personal de salud está expuesto y enferma de TBC. Unas de las pocas evidencias a
Tabla 4. Distribución de casos de Tuberculosis en el personal de salud de un Hospital de Lima según servicios hospitalarios, 2006-2013.

\begin{tabular}{lrr}
\hline Servicios Hospitalarios & n & \% \\
\hline Hospitalización de Especialidades & 17 & 30,4 \\
Mantenimiento y Limpieza & 9 & 16,1 \\
Hospitalización de Medicina & 7 & 12,5 \\
Emergencia & 7 & 12,5 \\
Servicios de Apoyo & 5 & 8,9 \\
Administración & 4 & 7,1 \\
Consultorios Externos & 3 & 5,4 \\
Laboratorio y Radiología & 2 & 3,6 \\
Unidad de Cuidados Críticos & 1 & 1,8 \\
Sala de Operaciones & 1 & 1,8 \\
Total & 56 & 100,0 \\
\hline
\end{tabular}

parte de nuestro estudio, son las investigaciones de Danilla et al (12) en el Hospital Nacional Arzobispo Loayza, entre 1999 y el 2003, donde encuentra 42 casos de TBC en el personal de salud; y de Accinelli et al (13) en el Hospital Nacional Cayetano Heredia, entre 1994 y el 2007 donde reporta otros 159 casos. Este último estudio además de lo ya mencionado, identifica que del 92,0\% de los médicos entrevistados que trataban pacientes con TBC activa; solamente el $7,4 \%$ de ellos utilizaban respirador al examinar dichos pacientes.

En comparación con el estudio de Danilla et al (12), hay diferencia en el grupo ocupacional más afectado (internos de Medicina 21,4\%), lo que no se refleja en nuestro estudio, en el que se observó que los principales grupos que afectados fueron los residentes de Medicina y los técnicos de enfermería; hallazgo similar al encontrado por Accinelli et al (13), donde los más afectados son los residentes de Medicina $(16,4 \%)$.

Así mismo, nuestra investigación muestra que los principales tipos de TBC que afectan a los trabajadores de salud son la TBC pulmonar sensible, TBC pulmonar MDR, seguidos por la TBC pleural, siendo el primero y el tercero los más frecuentes tanto en el estudio del Hospital Nacional Arzobispo Loayza con cifras de $69,0 \%$ y $8,2 \%$, como en el del Hospital Nacional Cayetano Heredia con $76,7 \%$ y $15,1 \%$; respectivamente. Es más, la tasa de letalidad en nuestro estudio fue $1,8 \%$, similar al 2,4\% encontrado por Danilla et al (12). 
Llama nuestra atención, la presencia de trabajadores de salud con TBC con BK negativo, raros a nivel mundial (14), pero que parece ser un patrón en los casos de TBC en los trabajadores de salud. Esto es importante mencionar porque tanto en los dos estudios anteriores como en el nuestro, alrededor de la mitad tuvo BK negativo, obligando a realizar el diagnóstico en base a la clínica y a los hallazgos radiológicos.

Por otro lado, es rescatable que del 2008 al 2012 se haya trabajado para disminuir los casos de trabajadores de salud con TBC, existiendo solo 2 casos en el 2012 a diferencia de años anteriores, en los cuales se llegó hasta 10 casos registrados. Esto demuestra que se ha estado intentando cumplir con la Visión del Programa de Control de Tuberculosis del HNHU: "Garantizar la detección, diagnóstico precoz, así como el tratamiento apropiado, oportuno, gratuito y supervisado de las personas afectadas con TBC en todos los servicios del hospital, brindando atención integral de calidad, con la finalidad de disminuir la morbi-mortalidad por TBC y sus repercusiones sociales y económicas" $(15,16)$.

Así mismo, se refiere que esta reducción se logró gracias al inicio del Programa de Control de Tuberculosis (PCT), a la instalación de filtros HEPA (High Efficiency Particulate Air) (17), de luz ultravioleta $(18,19)$ y a un estricto manejo de la bioseguridad y ventilación (20-22), lo cual genera una protección del $80 \%$ frente a bacterias y virus.

No obstante, en el 2013 ocurrió un incremento súbito a 10 casos registrados de $\mathrm{TBC}$ en el personal, lo cual fue debido al no uso de respiradores en los trabajadores de salud, según consta en el Informe anual de TBC nosocomial del Área de Salud Ocupacional del HNHU.

La principal limitación de la investigación es el pobre registro en el Perú de los casos de TBC, no porque el daño no esté, sino porque consideran a la TBC una enfermedad estigmatizante, y para evitar el rechazo social, se generan subregistros entre el personal expuesto (23), significando que tal vez existan cifras mayores a las reportadas. Esto se explicaría con el hecho de que muchos trabajadores de salud son diagnosticados de TBC en los consultorios del HNHU, pero por el mismo hecho de querer seguir laborando, esconden su situación de enfermedad y buscan otros hospitales como son los de ESSALUD o los del Sistema Metropolitano de la Solidaridad (SISOL) para realizar su tratamiento anti TBC, lo cual deja entre ver que quizás el número de trabajadores de salud del
HNHU con TBC pueda ser mayor al encontrado.

Otra limitación es el ser un estudio retrospectivo y de fuentes secundarias como lo son las historias clínicas, en las cuales muchas veces no se consignan los datos necesarios para el estudio.

En conclusión, los resultados del estudio describen epidemiológica y clínicamente a trabajadores de salud con TBC: más alta frecuencia en 2007 y 2013, con antecedente de TBC previa, la mayoría médicos residentes, $\mathrm{BK}$ negativo, con compromiso pulmonar, con TBC Pulmonar sensible y del servicio de Hospitalización de Especialidades. La mayoría recibió Esquema I y curaron en el mayor porcentaje.

A modo de recomendación, debe ser prioritario enfrentar el problema de la TBC en los trabajadores de salud, puesto que tanto las inadecuadas condiciones del ambiente hospitalario sumadas al no uso de medidas de bioseguridad, conllevarán a que el número de casos continúe elevándose. He ahí que se sugiere mayor rigurosidad en el monitoreo y control tanto de las medidas que tiene que tomar el propio trabajador hospitalario, como las que debe ejecutar el propio hospital para protección y disminución del riesgo de contraer TBC por sus trabajadores de salud.

\section{Declaración de finaenciamiento y conflictos de intereses}

El estudio fue financiado por los autores. Todos los autores declaran no tener conflicto de interés.

\section{Correspondencia}

\section{Mayron David Nakandakari Gómez}

Domicilio: Mz. A5 Lot. 10 AAHH. Cerro Candela. San Martín de Porres. Lima, Perú. Teléfono 51- 1- 7963569; 51-959727503

Correo electrónico: mdngmedicinaupsjb@hotmail. com

\section{REFERENCIAS BIBLIOGRÁFICAS}

1. Menzies D, Fanning A. Tuberculosis among health care workers. N Engl J Med. 1995; 332(2): 92-98.

2. Echanove J, Granich R. Occupational transmission of Mycobacterium tuberculosis to health care workers in a university hospital in Lima, Perú. Clin Infect Dis. 2001; 33:589-96.

3. Ministerio de Salud del Perú. Control de infecciones de tuberculosis en establecimientos de Salud. Módulo 
de capacitación. Lima: MINSA; 2005. RM-013.

4. Bonifacio N, Saito M. High risk for tuberculosis in hospital physicians, Peru. Emerg Infect Dis. 2002; 8(7):747-8

5. Sanghavi D, Gilman R, Lescano A, Checkley W, Cabrera L. Hyperendemic pulmonary tuberculosis in a Peruvian shantytown. Am J Epidemiol. 1998; 148:384-9.

6. Donayres L, Céspedes M, Valencia P, Salas J, Luna M. Peste neumónica primaria con transmisión intra hospitalaria en La Libertad, Perú 2010. Rev Perú Med Exp Salud Pública. 2010; 27(3):326-36.

7. Ministerio de Salud del Perú. Evaluación y calificación de la invalidez por accidentes de trabajo y enfermedades profesionales [documento técnico]. Lima: MINSA; 2011. RM-069.

8. Jensen P, Lambert L. Guidelines for preventing the transmission of Mycobacterium tuberculosis in health-care settings. MMWR Recomm Rep. 2005; 54(RR-17): 1-141.

9. Harries A, Maher D, Nunn P. Practical and affordable measures for the protection of health care workers from tuberculosis in low-income countries. Bull World Health Organ. 1997; 75(5):477-89.

10. Fica A, Cifuentes M. Tuberculosis en el personal de salud. Revista Chilena Infectol. 2008; 25:243-55.

11. Fica A, Ramonda P. Tuberculosis en el personal de salud del Servicio de Salud Metropolitano Sur de Santiago, Chile. Rev Chilena Infectol. 2009; 26(1): 34-38.

12. Danilla D, Gave J. Tuberculosis Ocupacional en un Hospital General de Lima, Perú. Revista de la Sociedad de Neumología. 2005; 49 (2): 101-105.

13. Accinelli R, Noda J, Bravo E, Galloso M, López L. Enfermedad tuberculosa entre trabajadores de salud. Acta Méd Peruana. 2009; 26 (1):35-47.

14. National Tuberculosis Controllers Association; Centers for Disease Control and Prevention (CDC). Guidelines for the investigation of contacts of persons with infection tuberculosis. Recommendations from the National Tuberculosis Controllers Association and CDC. MMWR Recomm Rep. 2005; 54(RR-15): $1-47$.

15. Ministerio de Salud del Perú. Norma Técnica N. ${ }^{\circ}$ 025: Actualización en la atención de pacientes con tuberculosis multidrogorresistente (TB MDR). Lima: MINSA; 2005.

16. Ministerio de Salud del Perú. Situación de la tuberculosis en el Perú. Informe 2011. Lima: MINSA; 2011.

17. Bonilla C. Situación de la tuberculosis en el Perú. Acta Med Perú. 2008; 25(3): 163-70.

18. Huaroto L, Espinoza M. Recomendaciones para el control de la transmisión de la tuberculosis en los hospitales. Rev Perú Med Exp Salud Publica. 2009; 26(3):364-9

19. Qian Y, Willeke K. Performance of N95 respirators: filtration efficiency for airborne microbial and inert particles. Am Ind Hyg Assoc J. 1998; 59(2): 128-32.

20. Ostrsoky L, Rangel. Tuberculosis en trabajadores de la salud: importancia de los programas de vigilancia y control. Salud Pública de México. 2000; 42:48-52.

21. Escombe A, Oeser C. Natural ventilation for the prevention of airborne contagion. PloS Med. 2007; 4(2): e68.

22. Joshi R, Reingold A. Tuberculosis among health care workers in low- and middle income countries: a systematic review. PLoS Med. 2006; 3(12):e494.

23. Cascante J, Hueto J. Tuberculosis como enfermedad ocupacional. An Sist Sanit Navar. 2005; 28(Supl. 1):107-15.

Recibido: 22/11/2013

Aceptado: 01/07/2014 